\title{
Direct Evidence for Calcineurin Binding to the Exon-7 Loop of the Sodium-Bicarbonate Cotransporter NBCn I
}

\author{
Harindarpal S. Gill1,2® ${ }^{\bowtie}$, Eric D. Roush³, Lauren Dutcher ${ }^{4}$, Samir Patel ${ }^{2}$ \\ 1. Department of Medicine, The George Washington University, Washington, DC 20052 \\ 2. Division of Renal Diseases and Hypertension, The George Washington Medical Faculty Associates, Washington, DC 20037 \\ 3. GE Healthcare, Piscataway, NJ 08554 \\ 4. Hospital of University of Pennsylvania, Philadelphia, PA 19104
}

$\bowtie$ Corresponding author: Harindarpal S. Gill, The George Washington University \& The GW Medical Faculty Associates, Department of Medicine, Division of Renal Diseases \& Hypertension, 2300 I (eye) Street NW, Ross Hall Room 436B, Washington, DC 20052. hgill@gwu.edu; Tel: 202-994-4587; Fax: 202-994-4538

( ) Ivyspring International Publisher. This is an open-access article distributed under the terms of the Creative Commons License (http://creativecommons.org/ licenses/by-nc-nd/3.0/). Reproduction is permitted for personal, noncommercial use, provided that the article is in whole, unmodified, and properly cited.

Received: 2014.04.29; Accepted: 2014.06.12; Published: 2014.07.04

\begin{abstract}
The $\mathrm{NaHCO}_{3}$ cotransporter $\mathrm{NBCnI}$ plays a role in neutralizing intracellular acid loads at the basolateral membrane in cells of the medullary thick ascending limb (mTAL). Calcineurin inhibitors $(\mathrm{Cn}-\mathrm{Is})$ are known to both downregulate NBCnI expression in the distal nephron and cause renal tubular acidosis (RTA), a risk factor for nephrocalcinosis and nephrolithiasis. In this report, we provide a new perspective on concurrent studies of NBCnl in various tissues by using cell-free binding assays to investigate the interaction of NBCn I with the calcineurin $(\mathrm{Cn})$ isoform PPP3CA. Surface plasmon resonance (SPR) analyses show that the protein domain Exon 7 (translated from cassette II of NBCnI) binds $\mathrm{Cn}$ with an equilibrium dissociation constant $\left(\mathrm{K}_{\mathrm{D}}\right)$ of $30+/-15 \mathrm{~nm}$. Linked-reaction tests suggest that the binding involves a conformational change. Nested PCR reactions were used to show that NBCn I-Exon 7 splice variants with alternative $\mathrm{N}$-termini regions are expressed in the kidney, as well as other tissues. Additionally, we discuss NBCnI-Exon 7 implication in acid-base balance and calcium crystallization in the kidney.
\end{abstract}

Key words: bicarbonate, transporters, calcineurin, renal physiology, surface plasmon resonance

\section{Introduction}

Bicarbonate $\left(\mathrm{HCO}_{3}{ }^{-}\right)$transport is critical for acid-base balance in normal tissue function throughout the body [1]. The $\mathrm{Na}^{+}$-coupled $\mathrm{HCO}_{3}^{-}$cotransporters, part of the solute carrier 4 (SLC4) family, are integral membrane proteins responsible for mediation of $\mathrm{Na}^{+}$ and $\mathrm{HCO}_{3}^{-}$by both reabsorption and secretion [2,3]. A structural analysis of all SLC4 family members illustrates the existence of three distinct domains: (i) a large cytoplasmic $\mathrm{N}$-terminus $(\mathrm{Nt})$ that contains one variable region at the extreme $\mathrm{Nt}(\mathrm{V} 1)$, and another in the middle (V2); (ii) a transmembrane domain (TMD) that spans the membrane up to 14 times; and (iii) a small cytoplasmic C-terminus ( $\mathrm{Ct}$ ). In the kidney, the electroneutral $\mathrm{Na}^{+} / \mathrm{HCO}_{3}{ }^{-}$cotransporter NBCn1
(SLC4A7), located at the basolateral membrane of the thick ascending limb, uses the 1:1 cellular uptake of $\mathrm{Na}^{+}$and $\mathrm{HCO}_{3}^{-}$to neutralize the intracellular acid produced by the apical uptake of $\mathrm{NH}_{4}$ [4-6]. NBCn1 has an unusually long cytoplasmic $\mathrm{Nt}$ that is about half the length of the entire protein. The unusual length of the $\mathrm{Nt}$ is partially due to its V2 variable region, a 13-residue sequence called cassette I (cassette $\mathrm{A}$ in older nomenclature), and a unique 124-residue sequence called cassette II that consists of an entire exon (exon-7 in older nomenclature).

New perspectives on the physiological importance of NBCn1 have recently emerged. Studies by Liu et al. (2013) suggest that NBCn1 is expressed in a 
wide distribution of human and murine tissues with four cassettes (I, II, III, and IV) that may be spliced in or out, as well as alternative initial $\mathrm{Nt}$ sequences [7]. The Nt can begin with either an initial 16 amino-acid sequence starting with MEAD, or an initial $10 \mathrm{ami}-$ no-acid sequence starting with MERF [7, 8]. Results from Liu et al. (2013) obviate early studies in which the insertion of cassettes and alternative $\mathrm{Nt}$ sequences were suggested to be species dependent.

Recent studies have investigated tissue-specific expression of NBCn1 variants. Expression of Exon 7 is thought to be tissue-specific. Screening studies by Yang et al. (2009) demonstrated that NBCn1-Exon 7 is expressed in heart, lung, spleen, and testis [9]. Whereas a more recent study by Lui et al. (2013) found this variant is expressed in heart, liver, and skeletal muscle [7]. Both screening studies concluded with a lack of NBCn1-Exon 7 expression in the kidney.

The physiological importance of NBCn1 variants was recently illustrated in a report from Danielsen et al. (2013), which demonstrated that the NBCn1-Exon 7 splice variants play important roles in intracellular $\mathrm{pH}$ regulation in vascular smooth muscle cells [10]. This report included yeast two-hybrid assays to provide evidence that Exon 7 binds with calcineurin $(\mathrm{Cn})$, a $\mathrm{Ca}^{2+}$-calmodulin activated Ser/Thr phosphatase known to modulate many ion channels and transporters [11]. Interestingly, it is also known that $\mathrm{Cn}$ inhibitors (Cn-Is), such as FK506 (tacrolimus), commonly used in immunosuppression therapy following kidney transplants, induce transient metabolic acidosis, reduce NBCn1 expression, and cause distal renal tubular acidosis (RTA) [12].

Encouraged by the aforementioned yeast two-hybrid assay results, and also the known NBCn1 modulation caused by $\mathrm{Cn}$-Is, we set out to determine the relationship between $\mathrm{Cn}$ and NBCn1 in the kidney. In the present study, we quantitatively describe the affinity of Cn-Exon 7 binding, and also provide evidence that NBCn1-Exon 7 splice variants are expressed in the kidney. Additionally, we propose a mechanism linking cellular $\mathrm{pH}$ regulation via NBCn1 to kidney stone formation.

\section{Materials and Methods}

\section{Identification and cloning of $\mathrm{N}$-terminal NBCn I splice variants by nested PCR}

NBCn1 splice variants were amplified by nested PCR reactions using human cDNA libraries, having adaptor-ligated AP1-ends, from kidney, skeletal muscle, and liver tissues (Clontech, CA). The kidney RNA used for cDNA library construction was from whole kidney after dissecting out the adrenal glands and included nephrons, renal cortex, and renal pelvis
(Clontech, CA). The PCR reaction consisted of $1 x$ enzyme buffer, $0.4 \mathrm{mM}$ dNTP mixture, $1 \mu \mathrm{M}$ forward primer (see below), $1 \mu \mathrm{M}$ reverse primer (see below), $\sim 100$ ng cDNA library, and 5U PfuTurbo ${ }^{\mathrm{TM}}$ DNA polymerase (Stratagene, La Jolla, CA). (See SUPPLEMENTARY MATERIAL for primer sequences.) The program of the thermal-cycler consisted of: (i) 1 cycle at $94{ }^{\circ} \mathrm{C}$ for $30 \mathrm{sec}$; (ii) 35 cycles at $94{ }^{\circ} \mathrm{C}$ for $30 \mathrm{sec}, 55$ ${ }^{\circ} \mathrm{C}$ for $1 \mathrm{~min}$, either $68^{\circ} \mathrm{C}$ for $10 \mathrm{~min}$; and (iii) 1 cycle at $68{ }^{\circ} \mathrm{C}$ for $10 \mathrm{~min}$ and $4{ }^{\circ} \mathrm{C}$ thereafter. PCR products were run on a $1 \%$ agarose gel against DNA markers, 1-kb and 2-log (New England Biolabs, MA). The nested-PCR products were subcloned into the Zero-Blunt ${ }^{\mathrm{TM}} \mathrm{TOPO}$ vector according to manufacturers protocol (Invitrogen, CA) and transformed in DH5 $\alpha$ cells. Isolated colonies were screened for insert and sequenced.

\section{Subcloning, Expression \& Purification of Exon 7}

The gene corresponding to Exon 7 was amplified by PCR using a fished-out full-length NBCn1 clone starting with the amino-acids "MERF..." as template and the following primers: the forward primer contained an NcoI restriction site, which merged immediately into a glycine codon plus a six-histidine codon stretch that preceded the first post-Met codon of cassette II: 5'-CATGCCATGGGACATCATCATCATCAT CATGGGGAAGGCCTTTCAG-3'. The reverse primer contained a stop-codon after the 372th bp of cassette II and an Xhol site thereafter: 5'-CCGCTCGAGTTA CTGGAGTTAAGTCAAC-3'. Reaction mixtures for the polymerase chain reaction were made according to the manufacture's protocol for Cloned-Pfu ${ }^{\mathrm{TM}}$ and contained $1 \mathrm{x}$ supplied buffer, $100 \mathrm{ng}$ template, $1 \mu \mathrm{M}$ of each primer, and $0.4 \mathrm{mM}$ of each dNTP in a $50 \mu \mathrm{l}$ final volume. PCR reaction cycle is the same as described in Gill et al. [13]. DNA products were then subcloned into the NcoI and XhoI restriction sites of the pET15B bacterial expression vector (Invitrogen, $\mathrm{CA})$, thereby removing the company's encoding sequence for a cleavable histidine-tag within the linker region of the vector.

Exon 7 was expressed in the Escherichia coli strain Rossetta2 $^{\mathrm{TM}}$ (Invitrogen, CA). Bacterial cells were grown at $37^{\circ} \mathrm{C}$ in a $200-\mathrm{L}$ fermentor using LB media supplemented with $50 \mu \mathrm{g} / \mathrm{ml}$ ampicillin, $34 \mu \mathrm{g} / \mathrm{ml}$ chloramphenicol, and aerated with 120 liters per minute filtered air with the vessel pressure at 20 psi until the $\mathrm{OD}_{600}$ reached 1.0. Cells were then induced with $0.5 \mathrm{mM}$ IPTG, harvested after $2 \mathrm{~h}$, and visually checked for over-expression of a $\sim 18-\mathrm{kDa}$ product on a gel, using SDS-polyacrylamide gel electrophoresis (SDS-PAGE). Note that the predicted molecular weight is $15-\mathrm{kDa}$, but the amino-acid sequence con- 
tains an abundance of positively charged (arginine and histidine) residues that likely retards its mobility on SDS-PAGE yielding an observed wave-like band. Using $15 \mathrm{~g}$ of cells, the purification of Exon 7 of NBCn1 followed the protocol as described in Gill et al. for NtNBCe1-A [13]. Purified Exon 7 was confirmed by $\mathrm{N}$-terminal protein sequence protocols (Keck Facility, Yale, New Haven). Affinity purified bovine calcineurin from brain was purchased commercially (C1907-100UN, Sigma-Aldrich, MO), having purification procedures based on Klee et al [14].

\section{Surface Plasmon Resonance (SPR)}

Using a Biacore ${ }^{\mathrm{TM}} \mathrm{T} 100$ (GE Healthcare, NJ Piscataway), flow cells of a carboxymethylated-dextran sensor chip (CM-5) chip were washed with $50 \mathrm{mM}$ $\mathrm{NaOH}$ in parallel using a flow rate of $10 \mu \mathrm{l} / \mathrm{min}$ for 3 min. Exon 7 was attached by amine coupling to a flow cell by activating the carboxymethyldextran surface of the targeted flow cell with an injection of a 1:1 mixture of $0.05 \mathrm{M}$ N-hydroxysuccinimide (NHS)/ $0.4 \mathrm{M}$ N-ethyl-N'-(dimethylaminopropyl)-carbodiimide (EDC) then injecting Exon 7 in $10 \mathrm{mM}$ sodium acetate at $\mathrm{pH} 5.5$ over the flow cell. Remaining active esters were blocked by an injection of $1 \mathrm{M}$ ethanolamine, $\mathrm{pH}$ 8.5 for 7 minutes. Surface density of Exon 7 was controlled by varying activation contact time, Exon-7 contact time, and Exon-7 concentration. A control surface on another flow cell was activated and blocked using the same conditions, but no protein was injected over the activated surface. The running buffer was $10 \mathrm{mM}$ HEPES, pH 7.4, $150 \mathrm{mM} \mathrm{NaCl}, 0.05 \%$ surfactant P20 (HBS-P+). Serial-diluted (analyte) solutions of bovine $\mathrm{Cn}$ and bovine serum albumin (BSA) in HBS-P+ were sequentially passed over both flow-cells: three-fold dilutions of $\mathrm{Cn}$ and in a separate experiment of BSA were injected for 3 min over an Exon-7 coupled surface yielding $200 \mathrm{RU}$ and a control surface. After the last injection, dissociation was monitored for $5 \mathrm{~min}$. Each binding cycle was performed at room temperature with a constant flow rate of $30 \mu \mathrm{L} / \mathrm{min}$ for $3 \mathrm{~min}$. Kinetic rate constants determination were obtained after subtraction of the reference and the buffer signals. Experiments were repeated five times under similar conditions, varying with different Exon-7 surface densities ranging from 63 to 10,000 RU and/or a CM-4 chip. Note that the amino-acid sequence of bovine $\mathrm{CnA}$ that contains the binding region is $97.5 \%$ identical to human $\mathrm{CnA}$
(100\% except for a 10 residue insertion in the C-terminus.) In general, the sequences of both $\mathrm{Cn}$ subunits and heterodimeric quaternary structure are highly conserved from yeast to mammals [15]. The PPP3CA isoform is required for normal kidney development and function [16]. Both kinetic rate constants and linked reaction models were fitted using the Biacore T100 Evaluation Software. Factors that were used to assess goodness of fit include residuals (difference between fitted curve and raw data for each point of the fit), chi-square (average squared residual), standard error for each parameter derived from the fit, and an overall qualitative evaluation of the goodness of fit by eye.

\section{Results \& Discussion}

\section{Quantitative analysis of Exon 7-Cn binding}

The interaction between purified Exon 7 and $C n$ was investigated using surface plasmon resonance (SPR) assays performed with single-cycle kinetics, a method in which a series of analyte concentrations are injected over active and control surfaces in one binding cycle without surface regeneration. Results in FIG 1 demonstrate the binding specificity of Exon 7. Data presented in FIG 1A show that BSA does not bind to Exon 7, even when at significantly higher concentration $(5 \times)$ than $\mathrm{Cn}$. Reference- and buffer-subtracted data were subjected to a global analysis with a nonlinear regression model that was determined to best describe the experimentally observed interaction. A two-state reaction model, implying a conformational change, was fit to the data (FIG 1B) and used to calculate an affinity $\left(K_{\mathbf{D}}\right)$ in the submicromolar range for the Exon 7-Cn interaction. The fit parameters (one of three repeated experiments) are shown in TABLE 1. This type of model was supported by data from a linked-reaction test, shown in FIG 1C, in which a constant concentration of $\mathrm{Cn}$ is injected into the experimental apparatus for different contact times while changes in dissociation rate are observed. The significant changes in dissociation rate as a function of contact time are indicative of a linked-reaction model. The tight binding of $\mathrm{Cn}$ to Exon 7 is uncommon, as others have cited much lower affinities (typically in the $1 \mu \mathrm{M}$ to $1 \mathrm{mM}$ range) for $\mathrm{Cn}$ and its other partners [17]. The Exon 7-Cn binding experiments were repeated using a variety of surface densities, yielding comparable results.

Table I. Fit Parameters for Two-State Model

\begin{tabular}{|c|c|c|c|c|c|c|c|c|c|c|}
\hline $\begin{array}{l}\mathrm{K}_{\mathrm{D}} \\
(\mathrm{nM})\end{array}$ & $\mathrm{k}_{\mathrm{a} 1}(1 / \mathrm{Ms})$ & $\begin{array}{l}\text { Standard } \\
\text { error }\left(\mathrm{k}_{\mathrm{a} 1}\right)\end{array}$ & $\begin{array}{l}\mathrm{k}_{\mathrm{d} 1} \\
(1 / \mathrm{s})\end{array}$ & $\begin{array}{l}\text { Standard } \\
\text { error }\left(k_{\mathrm{d} 1}\right)\end{array}$ & $\mathrm{k}_{\mathrm{a} 2}(1 / \mathrm{s})$ & $\begin{array}{l}\text { Standard } \\
\text { error }\left(\mathrm{k}_{\mathrm{a} 2}\right)\end{array}$ & $\begin{array}{l}\mathrm{k}_{\mathrm{d} 2} \\
(1 / \mathrm{s})\end{array}$ & $\begin{array}{l}\text { Standard } \\
\text { error }\left(k_{\mathrm{d} 2}\right)\end{array}$ & $\mathrm{R}_{\max }(\mathrm{RU})$ & $\begin{array}{l}\text { Standard } \\
\text { error }\left(R_{\max }\right)\end{array}$ \\
\hline 63 & $2.8 \mathrm{E}+5$ & $8.9 E+3$ & 0.081 & 0.003 & 0.006 & $3.4 \mathrm{E}-5$ & 0.002 & $8.8 \mathrm{E}-6$ & 168.6 & 0.54 \\
\hline
\end{tabular}


A

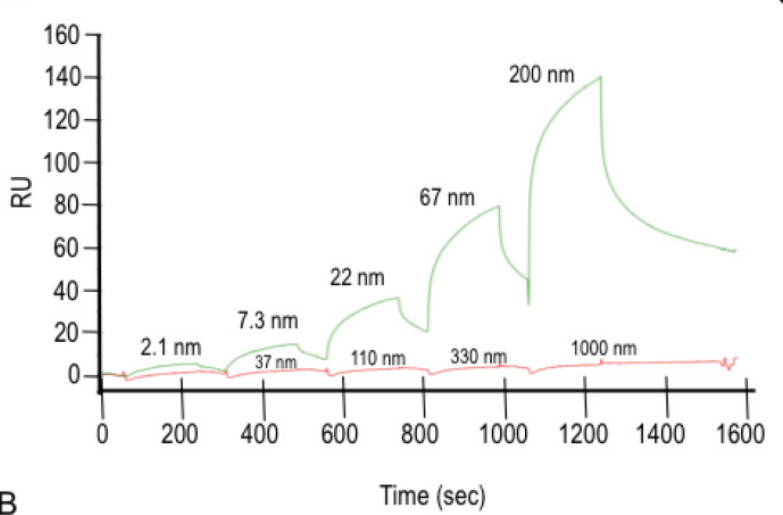

B

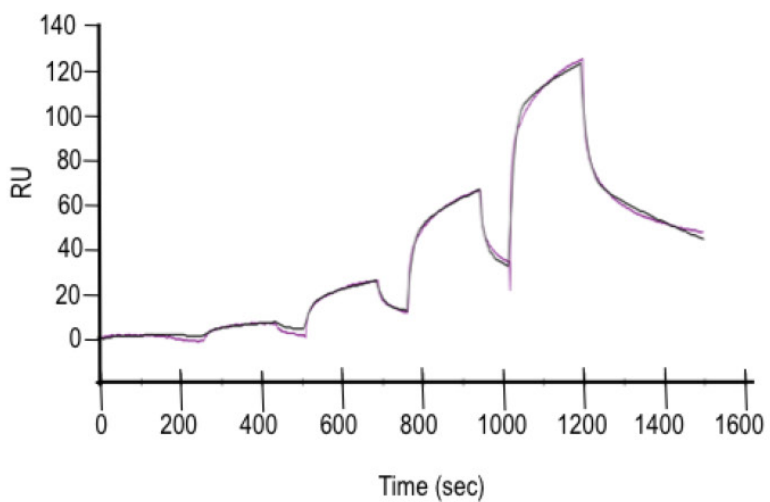

C

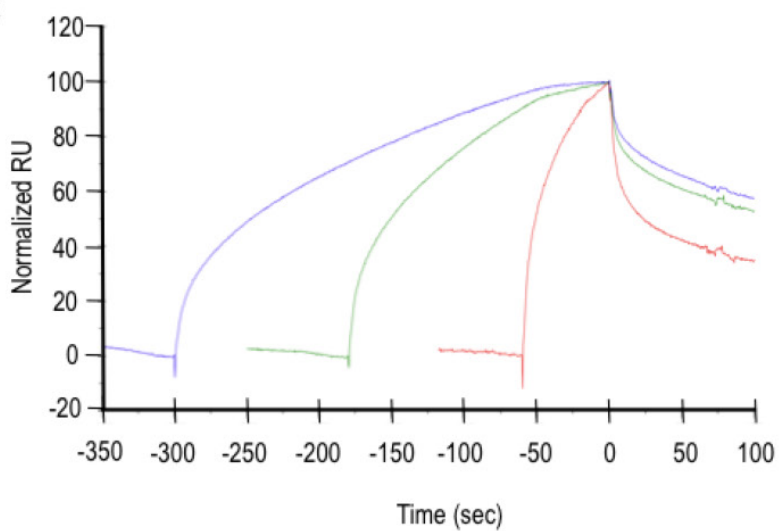

Figure I. Cell-free Exon 7-Cn binding experiments. A. Data is shown from experiments in which BSA was flowed over the Exon-7 surface (red curve) as a negative control, thus demonstrating the specificity of Exon 7 to $C n$ (green curve). $B$. A two-state reaction model $\left(A+B \leftrightarrow A B \leftrightarrow A B^{*}\right)$ was fitted to the Exon $7-C n$ binding curves. C. A linked-reaction test of the Exon 7-Cn interaction shows a significant difference in dissociation rates resulting from a change in contact time. Here, a solution of $66 \mathrm{nM} \mathrm{Cn}$ was injected for I min (red), $3 \mathrm{~min}$ (green), or $5 \mathrm{~min}$ (blue) over a 200 RU Exon 7 surface. Data have been reference-subtracted, aligned at the end of the $\mathrm{Cn}$ injection, and normalized ( 0 = baseline; $100=\mathrm{Cn}$ bound at end of injection).

\section{Tissue-specific expression of NBCn I-Exon 7 splice variants}

Given our results showing a high affinity interaction between $\mathrm{Cn}$ and Exon 7, and the known connection between $\mathrm{C} n$ and distal RTA, it seems logical to examine various tissues (including kidney) for expression of NBCn1-Exon 7 variants. Inspection of the human genome for exons encoding for alternative NBCn1 Nts (i.e., MEAD or MERF sequences) revealed the exon encoding for MEAD to be approximately 30,000 bp upstream of the exon encoding for MERF [8]. Similarly, inspection of the mouse genome revealed exons encoding the same alternative Nts. This is an important finding because it was previously thought that only an exon encoding for MEAD existed for this species.

After obtaining UTR sequences of NBCn1 from the human genome, PCR amplification was used to isolate 5'-clones of alternative $\mathrm{Nt}$ of $\mathrm{NBCn} 1$ splice variants from human cDNA libraries. Because NBCn1 levels were relatively low in tissues studied, nested PCR reactions were used for amplification. Similar to those reported in Liu et al. (2013), we found Nt variants consisting of two alternative sequences of the V1 region in combination with alternative cassettes of the $\mathrm{V} 2$ region. For the V1 region, the splice variants either started with MEAD, similar to those reported for rat NBCn1 [18], or with MERF. For the V2 region, both MEAD and MERF splice variants contained cassette I with or without cassette II (encoding Exon 7). FIG 2C summarizes in which tissues NBCn1 splice variants with and without cassette II were present.

These results could impact our understanding of NBCn1 physiology. We have shown that different tissues may exhibit differential expression of NBCn1 splice variants. Further investigations could include immunochemical analysis of various tissue types. However, probing for NBCn1 using only an antibody directed against the MERF-variant $\mathrm{Nt}$ would fail to detect NBCn1 splice variants beginning with MEAD. Although one might contemplate developing an antibody against MEAD, this peptide sequence closely resembles the $\mathrm{Nt}$ of another SLC4 family member, NCBE (SLC4A10). It is probable that the particular Nt sequence, along with the presence or absence of certain cassettes, affects NBCn1 physiology through influencing its binding partner and/or effects on TMD function. 

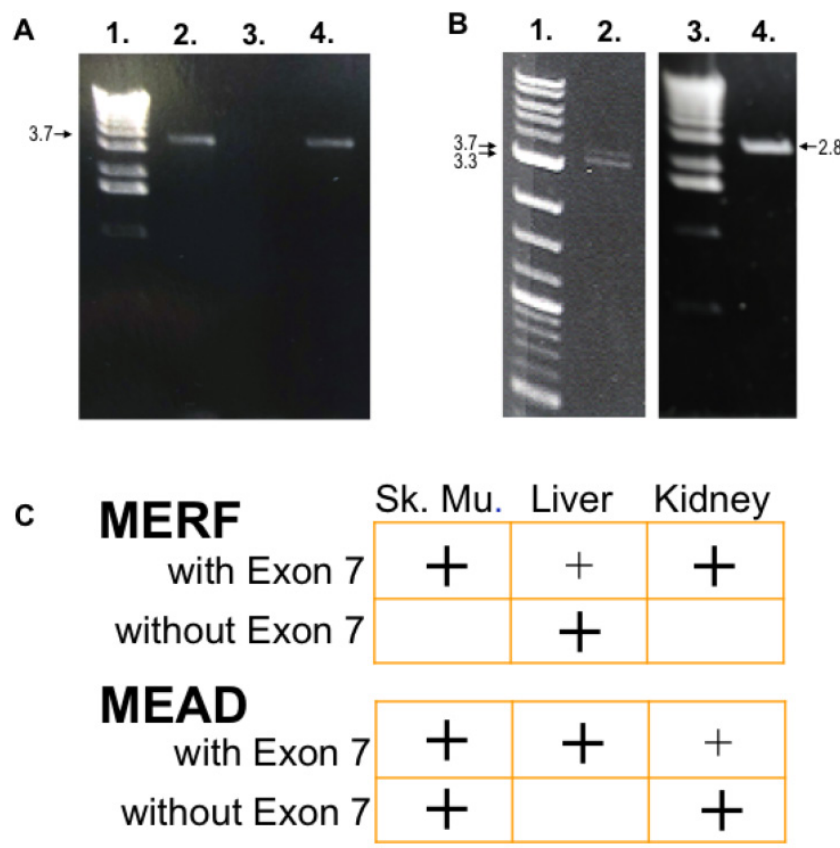

Figure 2. NBCn I-Exon 7 amplified from human tissues. A. Nested primers designed from the human genome upstream of the MERF initiation site were used in PCR amplification of cDNA from skeletal muscle (lane 2) and kidney (lane 4) libraries. Products were separated on a I\% agarose gel. The products are $\sim 3.7 \mathrm{kbp}$, as judged by comparison with the I-kb ladder (lane I). After sequencing, the bands were identified as genes encoding NBCnI containing Exon 7. The isolated kidney clone is identical to that of the NBC3 clone reported by the Kurtz group $[6,18]$, except for various point mutations and polymorphisms. No clones were amplified from liver (lane 3) with these particular primers. B. Examples of NBCnI clones with a MEAD initiation site are shown as amplified in skeletal muscle. Two bands ( $3.4 \mathrm{kbp}$ and $\sim 3.7 \mathrm{kbp}$; lane 2 ) encoding NBCn I were observed, as judged by comparison with the 2-log DNA marker (lane I). The upper band encodes for NBCnI with Exon 7, and the lower is without Exon 7. Similarly, an NBCn I-Exon 7 clone was amplified from liver (lane 4), and detected by internal gene-specific primers corresponding to an expected $\sim 2.8-\mathrm{kbp}$ product. C. A summary of the tissue distribution found for the Nt of NBCn I clones (with and without Exon 7) is shown in all experiments. There appears to be a complementary distribution of expression between $\mathrm{Nt}$-Exon 7 clones that begin with MEAD versus MERF. Large (easily detectable) and small (barely detectable) "+" signs indicate relative amplification intensity of signals using our primers.

\section{Link between NBCn I, Cn-Is, and kidney stones?}

Our discovery of NBCn1-Exon 7 expression in kidney tissue is an important finding, and suggests a potential mechanism for the development of Cn-I treatment-related RTA. Indeed, the loss of NBCn1-Exon 7 activity in the kidney, especially if promoted by the use of $\mathrm{Cn}$-Is, could be a factor contributing to kidney stone formation. However, the significance of NBCn1-Exon 7 in the kidney seems to have escaped adequate investigation. Early immunocytochemistry-based studies by the Kurtz group attempted to show that NBC3, an NBCn1 splice variant containing Exon 7, was present in rabbit collecting ducts [19]. In retrospect, because NBC3 was probed using an antibody against its $\mathrm{Ct}$, the presence of Exon 7 at the Nt was not verified.

Equally diminishing, previously performed mouse NBCn1 knockout studies also by the Kurtz group cited no apparent serum or urine electrolyte abnormalities, even though mouse models demon- strated ocular and auditory abnormalities due to sensory-neuron degeneration [20]. The details of urine- and serum-lab analyses were not presented in this report. It is worth noting that stone formation and distal RTA often yield serum and urine lab chemistries that appear normal [21], and urine citrate levels and are not routinely measured. Additionally, impaired acidification may not be apparent in normal physiological conditions, and only observable upon challenge with an acid load. This "incomplete" RTA may be diagnosed through administration of ammonium chloride in animal or human studies. A study making use of this protocol would have been a better tool for evaluation of NBCn1 knockout mice.

We also investigated interplay between NBCn1 and $\mathrm{Cn}$, a permissive phosphatase with multiple targets. A report from Mohebbi et al., which investigated connections between Cn-I, RTA, and NBCn1, found that inhibition of $\mathrm{Cn}$ resulted in lower NBCn1 expression, as well as lower expression of other acid-base transporters that are known to be associated with RTA (e.g., NHE3, NBCe1-A, and AE1). These results suggest $\mathrm{Cn}$-I causes an overall Type III RTA, rather than mere distal RTA. However, no direct binding between $\mathrm{Cn}$ and any of these transporters have been reported. Moreover, our sequence analysis fails to illuminate a typical putative Cn motif.

Further investigations into the role of NBCn1-Exon 7, as well as other acid-base cotransporters such as NBCe1-A, in the kidney may elucidate mechanisms of renal acid-base balance and calcium crystallization. NBCn1 is expressed in medullary thick ascending limb (mTAL) cells and is absent in proximal tubule (PT) cells, but its presence remains controversial in the collecting duct (CD) due to cross immunoreactivity to other SLC4s observed during immunochemical-based experiments [22, 23]. The existence of NBCn1-Exon 7 in renal epithelial cells remains to be demonstrated, and it is possible that the presence of Exon 7 in our commercial libraries could be derived from associated renal blood vesicles. In large, tissue-distribution studies of Exon 7 have been hampered by the lack of Exon 7 immunogenicity. If NBCn1-Exon 7 is truly found in mTAL epithelial cells, its downregulation would reduce bicarbonate uptake at the basolateral membrane. A recent model intro- 
duced by Odgaard et al. (2004) suggests that this action would decrease the neutralization of acid loads caused by the apical uptake of ammonium ions through the Rom K channel and the NaKCC cotransporter. The resulting intracellular acidosis could, in effect, lower the ammonium gradient across mTAL cells, inhibiting ammonia diffusion into the medullary interstitium. If downregulation of NBCn1-Exon 7 produces negative effects on ammonium recycling in the nephron, as well as intracellular acidosis of mTAL cells, this may explain the association between Cn-Is and RTA (and possibly hypocitraturia). Reduction of ammonia diffusion into the collecting tubule would result in impaired $\mathrm{H}^{+}$secretion from the distal nephron, leading to distal RTA that has been linked to Cn-I treatment. A connection between distal RTAs and hypocitraturia has already been described, and known to be involved in calcium crystallization and calcium-based stone formation [24, 25]. Even without systemic acidosis, intracellular acidosis may lead to increased renal tubular reabsorption of citrate and thus lower urinary citrate [26].

\section{Conclusion}

In this report, we successfully measured the interaction kinetics of the Exon-7 domain of NBCn1 and $\mathrm{Cn}$. Additionally, our results suggest that NBCn1-Exon 7 splice variants exist in the kidney, similarly to what has been found in other tissues. Finally, we suggest that NBCn1-Exon 7 expression in the kidney may play a significant role in systemic acid-base balance.

\section{Supplementary Material}

Primers for NBCn1 splice variants starting with MERF and MEAD. Additional nested-PCR amplification of NBCn1 with Exon 7 from kidney, liver and skeletal muscle cDNA libraries http://www.ijbs.com/v10p0771s1.pdf

\section{Acknowledgements}

We thank Drs. Timothy McCaffery, Sidney Fu, and Mark D. Parker for discussion, and Joseph Watson for help with the fermentor at Yale University. Award Number K01DK082646 (HSG) from the National Institute of Diabetes and Digestive and Kidney Diseases (NIDDK) of the National Institutes of Health (NIH) supported this project. The content is solely the responsibility of the author and does not necessarily represent the official views of the NIDDK or the NIH.

\section{Competing Interests}

The authors have declared that no competing interest exists.

\section{References}

1. Roos A, Boron WF. Intracellular pH. Physiological Reviews. 1981; 61: 296-434.

2. Choi I. SLC4A transporters. Curr Top Membr. 2012; 70: 77-103.

3. Romero MF, Fulton CM, Boron WF. The SLC4 family of $\mathrm{HCO}_{3}$-transporters. PflÅgers Archiv. 2004; 447: 495-509.

4. Odgaard E, Jakobsen JK, Frische S, Praetorius J, Nielsen S, Aalkjër C, et al. Basolateral Na+-dependent HCO3- transporter NBCn1-mediated HCO3influx in rat medullary thick ascending limb. Journal of Physiology, London. 2004; 555: 205-18.

5. Choi I, Aalkjër C, Boulpaep EL, Boron WF. An electroneutral sodium/bicarbonate cotransporter NBCn1 and associated sodium channel. Nature. 2000; 405: 571-5

6. Pushkin A, Abuladze N, Lee I, Newman D, Hwang J, Kurtz I. Cloning, tissue distribution, genomic organization, and functional characterization of NBC3, a new member of the sodium bicarbonate cotransporter family. Journal of Biological Chemistry. 1999; 274: 16569-75.

7. Liu Y, Qin X, Wang DK, Guo YM, Gill HS, Morris N, et al. Effects of optional structural elements, including two alternative amino termini and a new splicing cassette IV, on the function of NBCn1 (SLC4A7). J Physiol. 2013.

8. Gill HS, Dutcher LS, Parker MD, Boron WF. Identification and cloning of alternative N termini of human NBCn1. ProcPhysiolSoc. 2006; 3.

9. Yang HS, Cooper DS, Rajbhandari I, Park HJ, Lee S, Choi I. Inhibition of rat $\mathrm{Na}+(-) \mathrm{HCO} 3(-)$ cotransporter (NBCn1) function and expression by the alternative splice domain. Exp Physiol. 2009; 94: 1114-23

10. Danielsen AA, Parker MD, Lee S, Boron WF, Aalkjaer C, Boedtkjer E. Splice cassette II of $\mathrm{Na}+, \mathrm{HCO} 3(-)$ cotransporter $\mathrm{NBCn} 1$ (slc4a7) interacts with calcineurin A: implications for transporter activity and intracellular $\mathrm{pH}$ control during rat artery contractions. J Biol Chem. 2013; 288: 8146-55.

11. Liu L, Zhang J, Yuan J, Dang Y, Yang C, Chen X, et al. Characterization of a human regulatory subunit of protein phosphatase 3 gene (PPP3RL) expressed specifically in testis. Mol Biol Rep. 2005; 32: 41-5.

12. Mohebbi N, Mihailova $M$, Wagner CA The calcineurin inhibitor FK506 (tacrolimus) is associated with transient metabolic acidosis and altered expression of renal acid-base transport proteins. Am J Physiol Renal Physiol. 2009; 297: F499-509.

13. Gill HS, Boron WF. Expression and purification of the cytoplasmic N-terminal domain of the Na/HCO3 cotransporter NBCe1-A: structural insights from a generalized approach. Protein Expression and Purification. 2006; 49: 228-34.

14. Klee $\mathrm{CB}$, Crouch $\mathrm{TH}$, Krinks $\mathrm{MH}$. Calcineurin: a calcium- and calmodulin-binding protein of the nervous system. ProcNatlAcadSciUSA. 1979; 76: 6270-3.

15. Rusnak F, Mertz P. Calcineurin: form and function. Physiol Rev. 2000; 80: 1483-521.

16. Gooch JL, Toro JJ, Guler RL, Barnes JL. Calcineurin A-alpha but not A-beta is required for normal kidney development and function. Am J Pathol. 2004; 165: 1755-65.

17. Li H, Zhang L, Rao A, Harrison SC, Hogan PG. Structure of calcineurin in complex with PVIVIT peptide: portrait of a low-affinity signalling interaction. J Mol Biol. 2007; 369: 1296-306.

18. Cooper DS, Cucoranu D, Choi I. Role of the 123 amino acids deleted in the electroneutral sodium bicarbonate cotransporter (NBCn1). Neuroscience. 2004; Abstract.

19. Pushkin A, Yip KP, Clark I, Abuladze N, Kwon TH, Tsuruoka S, et al. NBC3 expression in rabbit collecting duct: colocalization with vacuolar $\mathrm{H}^{+}$-ATPase. American Journal of Physiology. 1999; 277: F974-F81.

20. Bok D, Galbraith G, Lopez I, Woodruff M, Nusinowitz S, BeltrandelRio H, et al. Blindness and auditory impairment caused by loss of the sodium bicarbonate cotransporter NBC3. Nature Genetics. 2003; 34: 313-9.

21. Rose BD, Post TW. Clinical physiology of acid-base and electrolyte disorders;5th ed. New York: McGraw-Hill. 2001.

22. Boedtkjer E, Praetorius J, Fuchtbauer EM, Aalkjaer C. Antibody-independent localization of the electroneutral $\mathrm{Na}+, \mathrm{HCO} 3-$ cotransporter NBCn1 (slc4a7) in mice. Am J Physiol Cell Physiol. 2008; 294: C591-603.

23. Parker MD, Boron WF. The divergence, actions, roles, and relatives of sodium-coupled bicarbonate transporters. Physiol Rev. 2013; 93: 803-959.

24. Stapenhorst L, Sassen R, Beck B, Laube N, Hesse A, Hoppe B. Hypocitraturia as a risk factor for nephrocalcinosis after kidney transplantation. Pediatr Nephrol. 2005; 20: 652-6.

25. Zuckerman JM, Assimos DG. Hypocitraturia: pathophysiology and medical management. Rev Urol. 2009; 11: 134-44.

26. Hamm LL. Renal handling of citrate. Kidney Int. 1990; 38: 728-35. 